\title{
Interpersonal, cognitive analytic and other integrative therapies versus other psychological therapies for depression
}

\author{
Vivien Hunot ${ }^{1}$, Theresa HM Moore ${ }^{1}$, Deborah Caldwell ${ }^{2}$, Philippa Davies ${ }^{1}$, Hannah Jones ${ }^{3}$, \\ Glyn Lewis $^{2}$, and Rachel Churchill ${ }^{1}$ \\ ${ }^{1}$ Academic Unit of Psychiatry, School of Social and Community Medicine, University of Bristol, \\ Bristol, UK. \\ ${ }^{2}$ School of Social and Community Medicine, University of Bristol, Bristol, UK. \\ ${ }^{3}$ Cochrane Schizophrenia Group, The University of Nottingham, Nottingham, UK
}

\section{Abstract}

This is the protocol for a review and there is no abstract. The objectives are as follows:

1. To examine the effectiveness and acceptability of all integrative therapies compared with all other psychological therapy approaches for acute depression.

2. To examine the effectiveness and acceptability of different integrative therapy models (IPT, CAT, psychodynamic-interpersonal therapy, CBASP, counselling) compared with all other psychological therapy approaches for acute depression.

3. To examine the effectiveness and acceptability of all integrative therapies compared with different psychological therapy approaches (psychodynamic, behavioural, humanistic, cognitive-behavioural, third wave CBT) for acute depression.

Copyright (C) 2012 The Cochrane Collaboration. Published by John Wiley \& Sons, Ltd.

Contact address: Rachel Churchill, Academic Unit of Psychiatry, School of Social and Community Medicine, University of Bristol, Oakfield House, Oakfield Grove, Bristol, Avon, BS8 2BN, UK. rachel.churchill@ccdan.org. rachel.churchill@bristol.ac.uk.

Editorial group: Cochrane Depression, Anxiety and Neurosis Group.

Publication status and date: Edited (no change to conclusions), published in Issue 7, 2012.

CONTRIBUTIONS OF AUTHORS Vivien Hunot provided theoretical and clinical expertise for designing this programme of 12 linked reviews of psychotherapies for depression, drawing from her training and clinical practice as a psychotherapeutic counsellor and cognitive behavioural therapist in NHS primary care settings. She worked on protocol development, developing a search strategy and compiling data extraction forms, and wrote the protocols for each review. Dr Hunot is responsible for writing and preparing this review. Along with Dr Rachel Churchill, she conducted the original review on which this programme is based.

Rachel Churchill conceived, designed, secured funding for, and is managing this programme of linked reviews. She has worked on all aspects of the development of this project, including building a review team, protocol development, developing a search strategy and compiling data-extraction forms. Along with Dr Vivien Hunot, she conducted the original review on which this programme is based. She is guarantor of the individual reviews in this programme of work.

Theresa Moore is managing the organisation of data for the 12 linked reviews of psychotherapies for depression including the search results, tracking of papers, and management of references for the project. She has developed the data collection forms. She designed the database and spreadsheets for data collection and has contributed to writing sections of the protocols and commented on text of the protocols.

Deborah Caldwell provided methodological and statistical advice for each of 12 linked protocols assessing the effects of different psychotherapies for depression. She contributed to the design of the data extraction form, drafted some sections of the protocols and commented on the protocol manuscripts. She designed the plan for the multiple treatment meta-analysis for the overview of reviews. Glyn Lewis provided a clinical perspective on 12 linked psychotherapies for depression protocols.

Philippa Davies contributed to the design of the review and development of the protocol.

Hannah Jones read and commented on the protocols.

DECLARATIONS OF INTEREST: None known. 


\section{BACKGROUND}

\section{Description of the condition}

Major depression is characterised by persistent low mood and loss of interest in pleasurable activities, accompanied by a range of symptoms including weight loss, insomnia, fatigue, loss of energy, inappropriate guilt, poor concentration and morbid thoughts of death (APA 2000). Somatic complaints are also a common feature of depression, and people with severe depression may develop psychotic symptoms (APA 2000).

Depression is the third leading cause of disease burden worldwide and is expected to show a rising trend over the next 20 years (WHO 2004; WHO 2008). A recent European study has estimated the point prevalence of major depression and dysthymia at $3.9 \%$ and $1.1 \%$ respectively (ESEMeD/MHEDEA 2004). As the largest source of non-fatal disease burden in the world, accounting for $12 \%$ of years lived with disability (Ustun 2004), depression is associated with marked personal, social and economic morbidity, loss of functioning and productivity and creates significant demands on service providers in terms of workload (NICE 2009). Depression is also associated with a significantly increased risk of mortality (Cuijpers 2002). The strength of this association, even taking account of confounders such as physical impairment, health-related behaviours and socio-economic factors, has been shown to be comparable to, or greater than, the strength of the association between smoking and mortality (Mykletun 2009).

\section{Description of the intervention}

Clinical guidelines recommend pharmacological and psychological interventions, alone or in combination, in the treatment of moderate to severe depression (NICE 2009). The prescribing of antidepressants has increased dramatically in many Western countries over the last 20 years, mainly with the advent of selective serotonin reuptake inhibitors and newer agents such as venlafaxine. Antidepressants remain the mainstay of treatment for depression in health care settings (Ellis 2004; NICE 2009).

Whilst antidepressants are of proven efficacy for the acute treatment of depression (Cipriani 2005; Guaiana 2007; Arroll 2009; Cipriani 2009; Cipriani 2009a; Cipriani 2009b), adherence rates remain very low (Hunot 2007; van Geffen 2009), in part due to patients' concerns about side effects and possible dependency (Hunot 2007). Furthermore, surveys consistently demonstrate patients' preference for psychological therapies over that of antidepressants (Churchill 2000; Riedel-Heller 2005). Therefore, psychological therapies provide an important alternative intervention for depressive disorders.

A diverse range of psychological therapies is now available for the treatment of common mental disorders (Pilgrim 2002). Psychological therapies may be broadly categorised into four separate philosophical and theoretical schools, comprising psychoanalytic/dynamic (Freud 1949; Klein 1960; Jung 1963), behavioural (Watson 1924; Skinner 1953; Marks 1982), humanistic (Maslow 1943; Rogers 1951; May 1961) and cognitive approaches (Lazarus 1971; Beck 1979). Each of these four schools contains a number of differing and overlapping psychotherapeutic approaches. 
In the 1960s, a discernible movement towards more integrated psychological therapy approaches began to emerge (Hollanders 2007). Also known as eclecticism, convergence and rapprochement, integrative psychological therapies are characterised by a dissatisfaction with single-school approaches and a desire to look beyond one school to see what can be learnt from other ways of thinking about psychotherapy and behaviour change (Norcross 2005); an approach described by Lazarus 2005 as 'breadth over depth'. Norcross 2005 identified a range of factors fostering development of integratism over the past two decades, including the proliferation of psychological therapies and a growing awareness that no one approach may be clinically adequate for all cases, together with increasing social-economic pressure to provide short-term problem-focused approaches.

There are considered to be four key operational 'routes' to integrating psychological therapy approaches, consisting of technical eclecticism, theoretical integration, the common factors approach and assimilative integration (Norcross 2005; Hollanders 2007). In technical, or 'systematic' eclecticism, practitioners use techniques, strategies and skills drawn from a range of different psychological therapy models. Techniqes are systematically matched with different stages in the therapeutic process, using a structured framework such as the multimodal approach (Lazarus 1989), or the 3-stage Skilled Helper model developed by Egan 1986. In theoretical integration, the goal is to create a framework that synthesises the most effective elements of two or more theoretical approaches and produces an emergent therapy that aims to offer more than the single therapies alone (Norcross 2005), as exemplified by the transtheoretical approach (that is, based on an analysis of different theories of psychotherapy) (Prochaska 1982). The common factors approach sets out to identify non-specific factors that are effective across each of the psychological therapies, focusing special attention on the therapeutic relationship (Orlinsky 2005), and to integrate these factors into a single model. In the assimilative approach, practitioners operate from one mainstream model, whilst adapting and integrating other techniques and strategies into the approach according to the needs of the individual patient, described by Yalom 2001 as creating 'a new therapy for each patient'.

As a manualised technically integrated model, interpersonal therapy (IPT) (Klerman 1984) is a key psychological therapy used in the treatment of depression. Theoretical integration approaches commonly used for depression include cognitive analytic therapy (CAT) (Ryle 1990), psychodynamic-interpersonal therapy (Shapiro 1990) and cognitive behavioural analysis system of psychotherapy (CBASP) (McCullough 1984). Counselling practitioners may use a range of different integrative approaches in treating depression, drawing from structured frameworks such as the skilled helper model or multimodal therapy, or based on assimilative integration (see Types of interventions section for a detailed description of each type of therapy).

\section{How the intervention might work}

"The broad and most basic goal of integrative therapy is for the therapist and client together to construct a therapy in which they will both be able to engage progressively in a fully collaborative process" (Hollanders 2007). There are no strategies or techniques that are intrinsically closed to the integrative therapist, although the way in which the therapy is 
constructed depends to some extent on whether the therapist considers the locus of integration to be predominantly external (relating to a specific approach), predominantly internal (in relation to the therapist's own values/beliefs) or somewhere between (constructed in a way that is determined by the emerging relationship between the therapist and client during therapy) (Hollanders 2007).

Integrative therapy approaches conceptualise and treat depression in different ways. For example, in IPT, depression is regarded as having three parts, consisting of symptoms, social/interpersonal life and personality. The therapist aims to understand the interpersonal context in which the depressive symptoms arose, and how they relate to the current social and personal context, with goals being to reduce symptomatology and to help the patient deal more effectively with the people and life situations associated with the onset of depression (Weissman 2007). In contrast, CAT conceptualises depression as a problematic 'coping procedure' rather than a set of symptoms (Llewelyn 2003). Therapy focuses on recognising how these problematic coping procedures originated (by examining the client's personal history and life experiences), and how they can be adapted and improved. Then, mobilising the clients' own strengths and resources, plans are developed to bring about change.

\section{Why it is important to do this review}

To date, evidence for the effectiveness of all integrative therapies for depression has been limited to systematic reviews investigating a single integrative approach, including IPT (Klerman 1984; de Mello 2005) and counselling in primary care settings (Bower 2006). Nevertheless, a survey of UK counsellors conducted 11 years ago (Hollanders 1999) suggested that a large proportion of practitioners placed themselves under a mainstream psychological therapy approach, whilst at the same time working integratively in clinical practice, and more recently, a cohort study conducted in UK primary care routine practice indicated that the proportion of counselling practitioners using an integrated approach approached $45 \%$ of those included in the study (Stiles 2008).

Clinical guidelines currently recommend interpersonal therapy as a first-line treatment for people with moderate to major depressive disorder (Ballenger 2001; NICE 2009) and counselling for mild to moderate depression (NICE 2009). Given the high prevalence of integrative approaches that continue to be used in UK clinical practice, and the prevailing lack of evidence on integrative therapies for depression, a comprehensive review of the effectiveness and acceptability of integrative psychological therapies for depression is required to inform and update clinical practice and future clinical guideline development. This review forms part of a programme of 12 reviews covering behavioural, cognitive behavioural, psychodynamic, interpersonal, cognitive analytic and other integrative, humanistic and mindfulness-based 'third wave' cognitive and behavioural psychological therapies, all compared with treatment as usual or with one another.

\section{OBJECTIVES}

1. To examine the effectiveness and acceptability of all integrative therapies compared with all other psychological therapy approaches for acute depression. 
2. To examine the effectiveness and acceptability of different integrative therapy models (IPT, CAT, psychodynamic-interpersonal therapy, CBASP, counselling) compared with all other psychological therapy approaches for acute depression.

3. To examine the effectiveness and acceptability of all integrative therapies compared with different psychological therapy approaches (psychodynamic, behavioural, humanistic, cognitive-behavioural, third wave CBT) for acute depression.

\section{METHODS}

\section{Criteria for considering studies for this review}

Types of studies-Randomised controlled trials (RCTs) will be eligible for inclusion in the review. Trials employing a cross-over design will be included in the review (whilst acknowledging that this design is rarely used in psychological therapy trials), using data from the first active treatment stage only. Cluster RCTs will also be eligible for inclusion.

Quasi-randomised controlled trials, in which treatment assignment is decided through methods such as alternate days of the week, will not be eligible for inclusion.

\section{Types of participants}

Participant characteristics: Studies of men and women aged 18 years will be included. A Cochrane review on psychotherapy for depression in children and adolescents $(<18$ years) has been undertaken separately and is soon to be published (Watanabe 2004). The increasing prevalence of memory decline (Ivnik 1992), cognitive impairment (Rait 2005) and multiple comorbid physical disorders/polypharmacy (Chen 2001) in individuals over 74 years may differentially influence the process and effect of psychological therapy interventions. Therefore, to ensure that older patients are appropriately represented in the review (Bayer 2000; McMurdo 2005) an upper age cutoff of $<75$ years will be used (when a study may have included individuals 75 , we will include it so long as the average age is $<75$ ), and a previously published Cochrane review on psychotherapeutic treatments for older depressed people (Wilson 2008) will be updated concurrently by the authors.

Setting: Studies may be conducted in primary care and community-based settings, or in secondary or specialist settings, and will include referrals as well as volunteers. Studies involving inpatients will be excluded. Studies that focus on specific populations - nurses, care givers, depressed participants at a specific work place - will be included if the participants all meet the criteria for depression.

Diagnosis: We will include all studies that focus on acute phase treatment of clinically diagnosed depression.

1. Studies adopting any standardised diagnostic criteria to define participants suffering from an acute phase unipolar depressive disorder will be included. Accepted diagnostic criteria include Feighner criteria, Research Diagnostic Criteria, DSM-III (APA 1980), DSM- III-R (APA 1987), DSM-IV-TR (APA 2000) or ICD-10 (WHO 1992) criteria. Earlier studies may 
have used ICD-9 (WHO 1978), but ICD-9 is not based on operationalised criteria, so studies using ICD-9 will be excluded from this category.

2. Mild, moderate and severe depressive disorders are all found in primary care (Mitchell 2009; Rait 2009; Roca 2009). In order to fully represent the broad spectrum of severity of depressive symptoms encountered by healthcare professionals in primary care, studies that used non-operationalised diagnostic criteria or used a validated clinician or self-report depression symptom questionnaire, such as Hamilton Rating Scale for Depression (Hamilton 1960) and Beck Depression Inventory (Beck 1961), to identify depression caseness based on a recognised threshold, will also be included. However, the influence of including this category of studies will be examined in a sensitivity analysis.

Accepted strategies for classifying mild, moderate and severe depression will be employed based, where possible, on those criteria used in the evidence syntheses underpinning the NICE 2009 guidelines for depression.

Studies focusing on chronic depression or treatment resistant depression, i.e. studies that listed these conditions as inclusion criteria, will be excluded from the review. Studies in which participants are receiving treatment to prevent relapse following a depressive episode (that is, where participants are not depressed at study entry) will also be excluded.

Studies of people described as 'at risk of suicide', or with dysthymia or other affective disorders such as panic disorder will be included if the participants meet criteria for depression as stated above, but will be excluded if not.

We will not include subgroup analyses of people with depression, selected out of people with mixed diagnoses, because such studies would be susceptible to publication bias (the authors reported such subgroup studies because the results were "interesting"). In other words we will include such studies only if the inclusion criteria for the entire study satisfied our eligibility criteria.

Comorbidity: Studies involving participants with comorbid physical or common mental disorders will be eligible for inclusion, as long as the comorbidity is not the focus of the study. In other words, we will exclude such studies which focused on depression among patients with Parkinson's disease or after acute myocardial infarction, but will accept such studies which may have included some subjects with Parkinson's disease or with acute myocardial infarction.

\section{Types of interventions}

Experimental interventions: Integrative approaches eligible for inclusion will be grouped into six main categories, according to the specific therapeutic principles and techniques described by trial authors, as follows:

1. Interpersonal therapy (IPT): IPT was developed in order to operationalise what was considered to be a set of helpful procedures commonly used in psychotherapy for depressed outpatients (Weissman 2007) for a series of treatment studies in depression conducted in the 
US (Elkin 1989; Frank 1990). Whilst described by the International Society for Interpersonal Psychotherapy (ISIPT) as having 'no specific theoretical origin' (isIPT [ND]), IPT draws in part from attachment theory (Bowlby 1980), and is based on the premise that depressive symptoms may be influenced strongly by the disruption of close personal attachments in four key domains of grief, role disputes, role transitions and interpersonal deficits (Weissman 2007). Within the therapeutic process, IPT uses techniques borrowed from other therapies such as cognitive-behaviour therapy and brief crisis intervention (isIPT [ND]), including clarification (seeking to obviate the patient's biases in describing interpersonal issues), role playing and communication analysis, together with supportive listening, encouragement of affect and use of the therapeutic relationship. Since the development of IPT in 1984, there has been ongoing debate over its potential categorisation as a time-limited psycho-dynamic psychotherapy (Markowitz 1998). Systematic reviews of psychodynamic therapy approaches for depression have obtained different findings according to its inclusion (Crits 1992; Anderson 1995) or exclusion (Svartberg 1991; Leichsenring 2004). Following detailed comparison of IPT and brief psychodynamic psychotherapy, Markowitz 1998 has concluded that despite overlaps and similarities, IPT is distinct from STPP. Given its use of CBT techniques, coupled with its psychodynamic features and atheoretical origins, IPT will be considered an integrative therapy for the purposes of this review, in accordance with technical integration principles.

2. Cognitive analytic therapy (CAT): CAT was developed by Ryle 1990 with the expressed purpose of providing an integrative therapy that would make use of the theoretical insights of CBT and the object relations theory (the process of developing a mind as one grows in relation to others in the environment) (Dunn 2007), within an accessible and time-limited model for application in the NHS for the treatment of depression. CAT focused attention on three common types of neurotic problems of snags (consequences anticipated as a result of change that themselves become obstacles to change), dilemmas ('either/ or' false dichotomies that restrict the range of choice, or 'if/then' false assumptions of association that similarly inhibit change), and traps (faulty perceptions of interpersonal options, for example, abuse or be abused, which lead to behaviour that confirms the perception) (Ryle 1979). CAT encourages clients to explore their own resources and develop the skills to change destructive patterns of behaviour and negative ways of thinking and acting.

The therapist works in collaboration with the client, focusing on changing maladaptive patterns of behaviour and teaching alternative strategies for coping. Attention is given to understanding the connections between childhood patterns, social input and their impact on the client as an adult. As a short term (16 weeks), structured and directive therapy, CAT uses prose reformulation letters to the client, summarising understandings, flow chart diagrams and questionnaires such as the CAT Psychotherapy File, together with CBT techniques such as diary keeping and rating sheets (Dunn 2007).

3. Psychodynamic-interpersonal therapy: This model is described as lying between traditional psycho-dynamic approaches and interpersonal psychotherapy (Guthrie 1999), and whilst theoretically derived from psychodynamic principles, also draws upon humanistic and interpersonal concepts. Originally called the conversational model of therapy, as the 
therapist and patient together develop a "mutual feeling language" and a relationship of "aloneness-togetherness" (Hobson 1985), it was manualised as psychodynamic-

interpersonal therapy by Shapiro 1990. It was designed for the therapy of patients whose symptoms and problems arose from difficulties or disturbances in interpersonal relationships, and can be used in its basic format to help individuals with a variety of symptomatic complaints, including depression. Key interlinking components are the provision of an exploratory rationale, developing a shared understanding, staying with/ focusing on difficult feelings, gaining insight, sequencing interventions and making changes (Guthrie 1999).

\section{Cognitive behavioural analysis system of psychotherapy (CBASP): CBASP}

(McCullough 1984) draws on many behavioural, cognitive, and interpersonal techniques used in other forms of psychotherapy. It teaches patients to focus on the consequences of their behaviour and to use a social problem-solving algorithm to address interpersonal difficulties and reduce depression symptoms. It is more structured and directive than interpersonal psychotherapy and differs from cognitive therapy by focusing primarily on interpersonal interactions (including those with therapists). In this type of psychotherapy, patients learn how their cognitive and behavioral patterns produce and perpetuate their interpersonal problems and learn how to remedy maladaptive patterns of interpersonal behaviour (Keller 2000).

5. Counselling: Counselling practitioners draw from a wide range of psychological therapy models, including person-centred, psychodynamic and cognitive behavioural approaches, applied discretely or integratively, according to the theoretical orientation of practitioners. According to Stiles 2008, $45 \%$ of patients treated in UK primary care routine practice are seen by a counsellor using an integrated approach that combines two or more of these models. Therefore, for the purposes of this review, counselling will be included in the integrative therapies category.

6. Motivational Interviewing: Motivational interviewing (MI) was developed with the intention of being used alongside other therapies, rather than as a stand-alone treatment (Miller 1983). Described as "client-centred therapy with a twist" (Arkowitz 2007a), MI is rooted in client-centred therapy principles (Rogers 1951), but also incorporates specific goals, in line with the structured 'skilled helper' model (Egan 1986), of reducing ambivalence about change and increasing intrinsic motivation to change (Miller 2002). MI uses four key principles, consisting of expressing empathy, developing discrepancy (increased awareness of differences between the client's behaviours and values), rolling with resistance (exploring the benefits and costs of making change) and supporting self-efficacy. For the purposes of using MI as a treatment for depression, Arkowitz 2007b sets out three levels of intervention. The first level is aimed at reducing major symptoms of depression using decisional balance strategies, in which a detailed exploration of reasons not to change is undertaken, while reasons to change are examined. At the second 'case formulation' level, the problems contributing to depression are identified and conceptualised in an integrative framework that draws from other diverse theoretical orientations. Arkowitz states that input from any reasonable theoretical orientation is appropriate. The third 'action' level is directed 
towards behaviour change, and again draws from other psychological therapy models in accordance with practitioners' preferred or selected approach.

7. Other integrative therapy approaches: Where studies evaluating an integrative therapy approach not listed above are identified, a post-hoc decision will be made about their inclusion in the review, with the impact of their inclusion examined in a sensitivity analysis (see Methods section), together with a more detailed description of the therapy in the Description of studies section.

Comparators: The control comparison will be all other types of psychological therapies, categorised as psychodynamic, behavioural, humanistic, cognitive behavioural and third wave CBT approaches.

1. Psychodynamic therapies: Grounded in psychoanalytic theory (Freud 1949), psychodynamic therapy (PD) uses the therapeutic relationship to explore and resolve unconscious conflict, through transference and interpretation, with development of insight and circumscribed character change as therapeutic goals, and relief of symptomatology as an indirect outcome. Brief therapy models have been devised by Malan 1963, Mann 1973 and Strupp 1984.

2. Behavioural therapies: Building on Skinner's theory of depression as an interruption in established sequences of health behaviour positively reinforced by the social environment (Skinner 1953), behavioural therapies focus attention on increasing access to pleasant events and positive reinforcers. The frequency of aversive events is decreased (Lewinsohn 1972) through monitoring of pleasant events, activity scheduling, social skills development and time management training (Hopko 2003).

2. Humanistic therapies: Contemporary models of humanistic therapies differ from one another somewhat in clinical approach, but all focus attention on the therapeutic relationship (Cain 2002), within which therapist 'core conditions' of empathy, genuineness and unconditional positive regard (Rogers 1951) are regarded as cornerstones to facilitate client insight and change.

4. Cognitive behavioural therapies: In cognitive behavioural therapy, therapists aim to work collaboratively with clients to understand the link between thoughts, feelings and behaviour, and to identify and modify unhelpful thinking patterns, underlying assumptions and idiosyncratic cognitive schema about the self, others and the world (Beck 1979). Cognitive change methods for depression are targeted at the automatic thought level in the first instance, and include thought catching, reality testing, task assignment and generating alterative strategies (Williams 1997). Behavioural experiments are then used to reevaluate underlying beliefs and assumptions (Bennett-Levy 2004).

5. Mindfulness-based 'third wave' cognitive and behavioural therapies: Third wave CBT approaches conceptualise cognitive thought processes as a form of 'private behaviour' (Hayes 2006; Hofmann 2008). Third wave CBT targets the individual's relationship with cognitions and emotions, focusing primarily on the function of cognitions such as thought 
suppression or experiential avoidance (an attempt or desire to suppress unwanted internal experiences, such as emotions, thoughts, and bodily sensations) (Hofmann 2008). A range of strategies, including mindfulness exercises, acceptance of unwanted thoughts and feelings, and cognitive diffusion (stepping back and seeing thoughts as just thoughts), are used to bring about change in the thinking process. Drawing from psychodynamic and humanistic principles, third wave CBT approaches also place great emphasis on use of the therapeutic relationship.

Format of psychological therapies: The psychological therapy intervention is required to be delivered through face to face meetings between the patient and therapist. Interventions in which face to face therapy is augmented by telephone or Internet-based support will be included in the review. Psychological therapy approaches conducted on either an individual or on a group basis will be eligible for inclusion. There is no limit to the number of sessions and we accept psychological therapy delivered in only one session.

Excluded interventions: Psychological therapy models based on social constructionist principles (that focus on the ways in which individuals and groups participate in the construction of their perceived social reality) including couples therapy, family therapy, solution-focused therapy, narrative therapy, personal construct therapy, neuro-linguistic programming and brief problem-solving (Watzlavick 1974) will be excluded. These therapies work with patterns and dynamics of relating within and between family, social and cultural systems in order to create a socially constructed framework of ideas (O'Connell 2007), rather than focusing on one individual's reality. Previously published Cochrane reviews on couples therapy for depression (Barbato 2006) and family therapy for depression (Henken 2007) will be updated concurrently.

Studies of long-term, continuation or maintenance therapy interventions designed to prevent relapse of depression or to treat chronic depressive disorders will be excluded from the review. Similarly studies of interventions designed to prevent a future episode of depression will be excluded.

Guided self-help, in which the practitioner provides brief face to face non-therapeutic support to patients who are using a self-help psychological therapy intervention, will be excluded as will bibliotherapy and writing therapies.

Psychological therapy that is provided wholly by telephone or over the Internet will not be eligible for inclusion.

Studies of dual modality treatments, in which patients are randomised to receive a combination of psychological and pharmacological treatments concurrently will only be included in the review if the study of interest compares two psychological models and both groups are prescribed the same concomitant pharmacological/placebo intervention.

Otherwise, these studies will be excluded from the current review, and will be examined in a separate programme of reviews on combination treatments for depression. Component or dismantling studies, in which the effectiveness of individual components of an interpersonal, cognitive analytic or other integrative therapies approach are investigated, will not be 
included. Data from these studies will be extracted and included in a separate overview of psychological therapies for depression, in which multiple treatments meta-analysis (MTM) will be used to compare the relative effectiveness of all psychotherapies, regardless of whether they have been directly compared in direct RCTs. If there is sufficient data, we will use the MTM model proposed inWelton 2009 to allow conclusions to be drawn regarding which components, or combination of components, are most effective at reducing depressive symptoms. See "Unit of analysis issues" for further detail on MTM.

Where the description of an intervention suggests it does not meet the inclusion criteria for an active psychological therapy approach, that study/study arm will be excluded from this review. If the intervention involved significant time spent with the patient, the review team will take a post hoc decision about whether it should be included as an attention placebo/ control in a linked review on interpersonal, cognitive analytic and other integrative therapies versus treatment as usual for depression.

\section{Types of outcome measures}

\section{Primary outcomes:}

1. Treatment efficacy: the number of patients who respond to treatment, based on changes on Beck Depression Inventory (BDI) (Beck 1961), Hamilton Rating Scale for Depression (HAM-D) (Hamilton 1960) or Montgomery-Asberg Depression Rating Scale (MADRS) (Montgomery 1979), or any other validated depression scale. Many studies define response by $50 \%$ or greater reduction on BDI, HAM-D etc but some studies define response using Jacobson's Reliable Change Index; we will accept the study authors' original definition. If the original authors report several outcomes corresponding with our definition of response, we will give preference to BDI for self-rating scale and HAM-D for observer-rating scale.

2. Treatment acceptability: the number of participants who drop out of psychological therapy treatment for any reason.

\section{Secondary outcomes:}

1. The number of patients who remit on treatment, based on the endpoint absolute status of the patients, as measured by Beck Depression Inventory (BDI) (Beck 1961), Hamilton Rating Scale for Depression (HAM-D) (Hamilton 1960) or Montgomery-Asberg Depression Rating Scale (MADRS) (Montgomery 1979), or any other validated depression scale. Examples of definitions of remission include 10 or less on BDI, 7 or less on HAM-D or 10 or less on MADRS; we will accept the study authors' original definition. If the original authors report several outcomes corresponding with our definition of response, we will give preference to BDI for self-rating scale and HAM-D for observer-rating scale.

2. Improvement in depression symptoms, based on a continuous outcome of group mean scores at the end of treatment using BDI, HAM-D, MADRS, or any other validated depression scale. 
3. Improvement in overall symptoms, using the Clinical Global Impressions Scale (CGI) (Guy 1976).

4. Improvement in anxiety symptoms, measured using a validated continuous scale, either assessor-rated, such as the Hamilton Anxiety Scale [HAM-A] (Hamilton 1959) or self-report, including the Trait subscale of the Spielberger State-Trait Anxiety Inventory (STAI-T) (Spielberger 1983) and the Beck Anxiety Inventory (BAI) (Beck 1988).

5. Adverse effects, such as completed suicides, attempted suicides and worsening of symptoms, where reported, will be summarised in narrative form.

6. Social adjustment, social functioning including the Global Assessment of Function (Luborsky 1962) scores, where reported, will be summarised in narrative form.

7. Quality of life, using validated measures such as the SF-36 (Ware 1993), HoNOS (Wing 1994) and WHOQOL (WHOQOL 1998), where reported, will be summarised in narrative form.

8. Economic outcomes (e.g. days of work absence/ability to return to work, number of appointments with primary care physician, number of referrals to secondary services, use of additional treatments) where reported, will be summarised in narrative form.

\section{Search methods for identification of studies Electronic searches}

CCDANCTR Registers: We will search two clinical trials registers created and maintained by the Cochrane Depression, Anxiety and Neurosis Group (CCDAN), the CCDANCTRStudies Register and the CCDANCTR-References Register. References to trials for inclusion in the Group's registers are collated from routine (weekly) searches of MEDLINE, EMBASE and PsycINFO, quarterly searches of the Cochrane Central Register of Controlled Trials (CENTRAL) and additional ad hoc searches of other databases (PSYNDEX, LILACS, AMED, CINAHL). These searches employ generic terms for depression anxiety and neuroses; together with sensitive (database specific) RCT filters.

References to trials are also sourced from international trials registers via the World Health Organisation's trials portal (http://apps.who.int/trialsearch/); drug companies; the handsearching of key journals, conference proceedings and other (non-Cochrane) systematic reviews and meta-analyses.

Details of the generic search strategies can be found in the 'Specialized Register' section of the Cochrane Depression, Anxiety and Neurosis Group's module text.

1. The CCDANCTR-Studies Register: The CCDANCTR-Studies Register contains over 11,000 trials for the treatment or prevention of depression, anxiety and neurosis. Each trial has been coded using the EU-Psi coding manual (as a guide) and includes information on intervention, condition, comorbidities, age, treatment setting etc. 
The studies register will be searched using the following search terms:

Condition $=($ depress $*$ or dysthymi $*)$ and Intervention $=(*$ therap $*$ or training $)$

2. The CCDANCTR-References Register: The CCDANCTR-References Register contains bibliographic records of reports of trials coded in the CCDANCTR-Studies Register together with several other uncoded references (total number of records $=24,500$ ). This register will be searched using a comprehensive list of terms for 'psychotherapies' as indicated in Appendix 1. Records already retrieved from the search of the CCDANCTR-Studies Register will be de-duplicated.

\section{Searching other resources}

1. Reference lists: The references of all selected studies will be searched for more published reports and citations of unpublished studies. Relevant review papers will be checked.

2. Personal communication: Subject experts will be contacted to check that all relevant studies, either published or unpublished, have been considered for inclusion.

\section{Data collection and analysis}

Selection of studies-Two review authors ( $\mathrm{RC}$ and $\mathrm{VH})$ will examine the abstracts of all publications obtained through the search strategy. Full articles of all the studies identified by either of the review authors will then be obtained and inspected by the same two review authors for trials meeting the following criteria:

1. Randomised controlled trial;

2. Participants have depression diagnosed by operationalised criteria;

3. Any integrative therapy approach (IPT, CAT, psychodynamic-interpersonal therapy or counselling), to include supportive or non-directive placebo control therapies, compared with any other psychological therapy approach.

Conflicts of opinion regarding eligibility of a study will be discussed with a third review author, having retrieved the full paper and consulted the authors if necessary, until consensus is reached. External subject or methodological experts will be consulted if necessary.

Data extraction and management-Data will be extracted by two review authors, with data from each study being extracted independently by two of these authors. Any disagreement will be discussed with an additional review author and where necessary, the authors of the studies will be contacted for further information.

Information relating to study population, sample size, interventions, comparators, potential biases in the conduct of the trial, outcomes including adverse events, follow-up and methods of statistical analysis will be abstracted from the original reports into specially designed paper forms then entered into a spreadsheet. 
Management of time points: Post-treatment outcomes and outcomes at each reported follow-up point will be summarised. Where appropriate and if the data allow, outcomes will be categorised as short term (up to 6 months post-treatment), medium term (7 to 12 months post-treatment) long term (longer than 12 months).

Assessment of risk of bias in included studies-Risk of bias will be assessed for each included study using the Cochrane Collaboration 'risk of bias' tool (Higgins 2008a). The following six domains will be considered:

1. Sequence generation: was the allocation sequence adequately generated?

2. Allocation concealment: was allocation adequately concealed?

3. Blinding of participants, personnel and outcome assessors for each main outcome or class of outcomes: was knowledge of the allocated treatment adequately prevented during the study?

4. Incomplete outcome data for each main outcome or class of outcomes: were incomplete outcome data adequately addressed?

5. Selective outcome reporting: are reports of the study free of suggestion of selective outcome reporting?

6. Other sources of bias: was the study apparently free of other problems that could put it at a high risk of bias? Additional items to be included here are therapist qualifications, treatment fidelity and researcher allegiance/conflict of interest.

A description of what was reported to have happened in each study will be provided, and a judgement on the risk of bias will be made for each domain within and across studies, based on the following three categories:
A. Yes (low risk of bias)
B. Unclear
C. No (high risk of bias).

Two independent review authors will assess the risk of bias in selected studies. Any disagreement will be discussed with a third review author. Where necessary, the authors of the studies will be contacted for further information. All risk of bias data will be presented graphically and described in the text. Allocation concealment will be used as a marker of trial quality for the purposes of undertaking sensitivity analyses.

\section{Measures of treatment effect}

Continuous outcomes: Where studies have used the same outcome measure for comparison, data will be pooled by calculating the mean difference (MD). Where different measures are used to assess the same outcome, data will be pooled with standardised mean difference (SMD) and 95\% confidence intervals calculated.

Dichotomous outcomes: These outcomes will be analysed by calculating a pooled odds ratio (OR) and 95\% confidence intervals for each comparison. Because ORs can be difficult 
to interpret, these pooled ORs will be converted to relative risks (RR) using the formula provided in The Cochrane Handbook (Higgins 2008) and presented in this form for ease of interpretation.

Unit of analysis issues-Multiple-arm studies (those with greater than two intervention arms) can pose analytical problems in pair-wise meta-analysis. For studies with more than two relevant active treatment arms data will be managed in this review as follows:

Continuous data: Means, SDs and number of participants for each active treatment group will be pooled across treatment arms as a function of the number of participants in each arm to be compared against the control group (Law 2003; Higgins 2008; Higgins 2008b).

Dichotomous data: Data from relevant active intervention arms will be collapsed into a single arm for comparison or data from relevant active intervention arms will be split equally between comparator arms.

Multiple treatment meta-analysis: One method which retains the individual identity of each intervention and allows multiple intervention comparisons to be made, without the need to lump or split intervention arms, is a multiple treatment meta-analysis (MTM) (Lu 2004; Caldwell 2005;Cipriani 2009b). MTM (also known as Mixed Treatment Comparison or Network Meta-analysis) refers to ensembles of trial evidence in which direct and indirect evidence on relative treatment effects are pooled. The objective of an MTM analysis is to combine all the available trial evidence into an internally consistent set of estimates while respecting the randomisation in the evidence. An MTM provides estimates of the effect of each intervention relative to every other, whether or not they have been directly compared in trials. One can also calculate the probability that each treatment is the most effective. We do not intend to use an MTM in this review, as we are unlikely to have sufficient data for the analysis. However, this review forms part of a series of 12 reviews which will contribute studies to an overview of reviews (Becker 2008;Higgins 2008b) in which MTM will be used as the main analytical strategy.

Dealing with missing data-Missing dichotomous data will be managed through intention to treat (ITT) analysis, in which it will be assumed that patients who dropped out after randomisation had a negative outcome. Best / worse case scenarios will also be calculated for the clinical response outcome, in which it will be assumed that dropouts in the active treatment group had positive outcomes and those in the control group had negative outcomes (best case scenario), and that dropouts in the active treatment group had negative outcomes and those in the control group had positive outcomes (worst case scenario), thus providing boundaries for the observed treatment effect. If there is a large amount of missing information then these best / worst case scenarios will be given greater emphasis in the presentation of the results.

Missing continuous data will be either analysed on an endpoint basis, including only participants with a final assessment, or analysed using last observation carried forward to the final assessment (LOCF) if LOCF data were reported by the trial authors. Where SDs are missing, attempts will be made to obtain these data through contacting trial authors. Where 
SDs are not available from trial authors, they will be calculated from $\mathrm{P}$ values, $\mathrm{t}$-values, confidence intervals or standard errors, where reported in articles (Deeks 1997).

Where the vast majority of actual SDs are available and only a minority of SDs are unavailable or unobtainable, a method used for imputing SDs and calculating percentage responders devised by Furukawa and colleagues (Furukawa 2005; Furukawa 2006) will be used. Where this method is employed, data will be interpreted with caution, taking account of the degree of heterogeneity observed. A sensitivity analysis will also be undertaken to examine the effect of the decision to use imputed data.

Where additional figures are not available or obtainable, and it is not deemed appropriate to use the Furukawa method described above, the study data will not be included in the comparison of interest.

Assessment of heterogeneity-Statistical heterogeneity will be formally tested using the chi $^{2}$ test, which provides evidence of variation in effect estimates beyond that of chance. Since the $\mathrm{chi}^{2}$ test has low power to assess heterogeneity where a small number of participants or trials are included, the $\mathrm{P}$ value will be conservatively set at 0.1 . Heterogeneity will also be quantified using the $\mathrm{I}^{2}$ statistic, which calculates the percentage of variability due to heterogeneity rather than chance. We expect, a priori, that there will be considerable clinical heterogeneity between studies and so $\mathrm{I}^{2}$ values in the range of $50 \%$ to $90 \%$ will be considered to represent substantial statistical heterogeneity and will be explored further. However, the importance of the observed $\mathrm{I}^{2}$ will depend on the magnitude and direction of treatment effects and the strength of evidence for heterogeneity (Higgins 2003; Deeks 2008). Forest plots generated in RevMan 5 now also provide an estimate of tau ${ }^{2}$, the between-study variance in a random-effects meta-analysis. To give an indication of the spread of true intervention effects we will use the tau ${ }^{2}$ estimate to form an approximate range of intervention effects using the method outlined in section 9.5.4 of the Cochrane Handbook for Systematic Reviews of Interventions (Deeks 2008). This will be undertaken for the primary outcomes only.

Assessment of reporting biases-As far as possible, the impact of reporting biases will be minimised by undertaking comprehensive searches of multiple sources (including trial registries), increasing efforts to identify unpublished material, and including nonEnglish language publications.

We will also try and identify outcome reporting bias in trials by recording all trial outcomes, planned and reported and noting where there are missing outcomes. Where we find evidence of missing outcomes, we will attempt to obtain any available data direct from the authors.

Where sufficient numbers of trials allow for a meaningful analysis, funnel plots will be constructed to establish the potential influence of reporting biases and small study effects.

Data synthesis-Given the potential heterogeneity of psychological therapy approaches for inclusion, together with the likelihood of differing secondary comorbid mental disorders in the population of interest, a random-effects model will be used in all analyses. 


\section{Subgroup analysis and investigation of heterogeneity}

\section{Clinical heterogeneity:}

1. Baseline depression severity: the severity of depression on entering the trial is expected to have an impact on outcome. Heterogeneity analyses will categorise baseline severity as mild, moderate or severe.

2. Number of sessions: there are likely to be differences in the numbers of therapy sessions received and this is expected to affect treatment outcome. Numbers of sessions will be categorised as 1 to 7 sessions, 8 to 12 sessions, 13 to 20 sessions and more than 20 sessions.

3. Type of comparison: the type of comparator used is likely to influence the observed effectiveness of the intervention. Where possible, comparators will be categorised according as psychodynamic, BT, humanistic, CBT and third wave CBT.

4. Strength of therapeutic alliance/perceived therapist empathy, based on validated measures such as the Barrett-Lennard Relationship Inventory (Barrett-Lennard 1986) or Working Alliance Inventory (Horvath 1986): where reported, this information will be summarised in narrative form.

\section{Sensitivity analysis:}

1. Fidelity to treatment: studies that have not assessed fidelity to the psychological therapy model(s) under evaluation through assessment of audio or videotapes of therapy sessions will be excluded

2. Study quality: allocation concealment will be used as a marker of trial quality. Studies that have not used allocation concealment will be excluded.

3. Trials where missing data has been imputed will be excluded.

4. Antidepressant treatment (naturalistic use; combination treatment used in both psychological therapy arms)

5. Trials included in the review following post-hoc decisions about their eligibility as interpersonal, cognitive analytic or other integrative therapeutic approach will be excluded.

\section{Acknowledgments}

\section{SOURCES OF SUPPORT}

Internal sources

- University of Bristol, UK.

External sources

- Department of Health, UK.

NIHR Programme Grant 


\section{Appendix 1. CCDANCTR references search}

Title, Abstract, Keywords = (depress* or dysthymi* $)$

and

Title, Abstract, Keywords $=((*$ therap* and $(($ acceptance* or commitment* $)$ or "activity scheduling" or alderian or art or aversion or brief or "client cent*" or cognitive or color or colour or "compassion-focused" or "compassion* focus*" or compassionate or conjoint or conversion or conversational or couples or dance or dialectic* or diffusion or distraction or eclectic or "emotion* focus*" or emotion-focus* or existential or experiential or exposure or expressive or family or focus-oriented or "focus oriented" or freudian or gestalt or group or humanistic or implosive or insight or integrative or interpersonal or jungian or kleinian or marital or metacognitive or meta-cognitive or milieu or morita or multimodal or multimodal or music or narrative or nondirective or non-directive or "non directive" or nonspecific or non-specific or "non specific" or "object relations" or "personal construct" or "person cent*" or person-cent* or persuasion or play or "pleas* event*" or primal or problem-focused or "problem focused" or problem-solving or "problem solving" or processexperiential or "process experiential" or psychodynamic or "rational emotive" or reality or "reciprocal inhibition" or relationship* or reminiscence or restructuring or rogerian or schema* or self-control* or "self control*" or "short term" or short-term or sex or "social effectiveness" or "social skill*" or socio-environment* or "socio environment*" or "solution focused" or solution-focused or "stress management" or supportive or time-limited or "time limited" or "third wave" or transference or transtheoretical or validation)) or abreaction or "acting out" or "age regression" or ((assertive* or autogenic or mind or sensitivity) and train*) or autosuggestion or "balint group" or ((behavior* or behaviour*) and (activation or therap* or treatment or contracting or modification)) or biofeedback or catharsis or cognitive or "mind training" or counsel* or "contingency management" or countertransference or "covert sensitization" or "eye movement desensiti*" or "crisis intervention" or "dream analysis" or "emotional freedom" or "free association" or "functional analys*" or griefwork or "guided imagery" or hypno* or imagery or meditation* or "mental healing" or mindfulness* or psychoanaly* or psychodrama or psychoeducat* or "psycho* support*" or psychotherap* or relaxation or "role play*" or "self analysis" or "self esteem" or "sensitivity training" or "support* group*" or therapist or "therapeutic technique*" or "transactional analysis")

\section{HISTORY}

Protocol first published: Issue 9, 2010

\section{WHAT'S NEW}

\begin{tabular}{lll}
\hline Date & Event & Description \\
\hline 13 June 2012 & Amended & Minor changes to methods \\
\hline
\end{tabular}




\section{Additional references}

Anderson 1995 . Anderson EM, Lambert MJ. Short-term dynamically oriented psychotherapy: a review and meta-analysis. Clinical Psychology Review. 1995; 15:503-14.

APA 1980 . American Psychiatric Association. Diagnostical and Statistical Manual of Mental Disorders (DSM-III). 3rd Edition. American Psychiatric Association; Washington DC: 1980.

APA 1987 . American Psychiatric Association. Diagnostical and Statistical Manual of Mental Disorders (DSM-III-R). 3rd Edition. American Psychiatric Association; Washington DC: 1987.

APA 1994 . American Psychiatric Association. Diagnostical and Statistical Manual of Mental Disorders (DSM-IV). 4th Edition. American Psychiatric Association; Washington DC: 1994.

APA 2000 . American Psychiatric Association. Diagnostical and Statistical Manual of Mental Disorders (DSM-IV-TR). 4th Edition. American Psychiatric Association; Washington DC: 2000.

Arroll 2009 . Arroll B, Elley CR, Fishman T, Goodyear-Smith FA, Kenealy T, Blashki G, Kerse N, MacGillivray S. Antidepressants versus placebo for depression in primary care. Cochrane Database of Systematic Reviews. 2009; (Issue 3) [DOI: 10.1002/14651858].

Balint 1972 . Balint, M.; Ornstein, PO.; Balint, E. Focal psychotherapy. Tavistock; London: 1972.

Ballenger 2001 . Ballenger JC, Davidson JR, Lecrubier Y, Nutt DJ. A Proposed Algorithm for Improved Recognition and Treatment of the Depression/Anxiety Spectrum in Primary Care. Prim Care Companion J Clin Psychiatry. 2001; 3(2):44-52. [PubMed: 15014615]

Barbato 2006 . Barbato A, D’Avanzo BBD. Marital therapy for depression. Cochrane Database of Systematic Reviews. 2006; (Issue 1) [DOI: 10.1002/14651858.CD004188.pub2].

Barrett-Lennard 1986 . Barrett-Lennard, GT. The relationship inventory now: Issues and advances in theory, method and use. In: Greenberg, LS.; Pinsof, W., editors. The Psychotherapeutic Process: A Research Handbook. Guildford Press; New York: 1986.

Bayer 2000 . Bayer A, Tadd W. Unjustified exclusion of elderly people from studies submitted to research ethics committee for approval: descriptive study. BMJ. 2000; 321(7267):992-3. [PubMed: 11039965]

Beck 1961 . Beck AT, Ward CH, Mendelsohn M, Mock J, Erbaugh J. An inventory for measuring depression. Archives of General Psychiatry. 1961; 4:561-71. [PubMed: 13688369]

Beck 1979 . Beck, AT.; Rush, AJ.; Shaw, BF.; Emery, G. Cognitive Therapy of Depression. Guildford Press; New York: 1979.

Beck 1988 . Beck AT, Epstein N, Brown G, Steer RA. An inventory for measuring clinical anxiety: psychometric properties. Journal of Consulting and Clinical Psychology. 1988; 56(6):893-7. [PubMed: 3204199]

Becker 2008 . Becker, LA.; Oxman, AD. Overviews of reviews. In: Higgins, JPT.; Green, S., editors. Cochrane Handbook for Systematic Reviews of Interventions. Version 5.0.2. The Cochrane Collaboration; 2008. Available from www.cochrane-handbook.org [updated September 2009]

Bennett-Levy 2004 . Bennett-Levy, J.; Butler, G.; Fennell, M.; Hackmann, A.; Mueller, M.; Westbrook, D. Oxford Guide to Behavioural Experiments in Cognitive Therapy. Oxford University Press; 2004.

Bortolotti 2008 . Bortolotti B, Menchetti M, Bellini F, Montaguti MB, Berardi D. Psychological interventions for major depression in primary care: a meta-analytic review of randomized controlled trials. General Hospital Psychiatry. 2008; 30(4):293-302. [PubMed: 18585531]

Bower 2006 . Bower PJ, Rowland N. Effectiveness and cost effectiveness of counselling in primary care. Cochrane Database of Systematic Reviews. 2006; (Issue 3) [DOI: 10.1002/14651858.CD001025.pub2].

Bowlby 1980 . Bowlby, J. Attachment and Loss. Vol. Vol 3. Hogarth Press; London: 1980. Loss: Sadness \& Depression.

Cain 2002 . Cain, DJ.; Seeman, J. Humanistic Psychotherapies: Handbook of Research and practice. American Psycholoigcal Association; Washington, DC: 2002.

Caldwell 2005 . Caldwell DM, Ades AE, Higgins JPT. Simultaneous comparison of multiple treatments: combining direct and indirect evidence. BMJ. 2005; 331:897-900. [PubMed: 16223826] 
Chen 2001 . Chen YF, Dewey ME, Avery AJ. Self-reported medication use for older people in England and Wales. J Clin Pharm Ther. 2001; 26(2):129-40. [PubMed: 11350536]

Churchill 2000 . Churchill R, Khaira M, Gretton V, Chilvers C, Dewey M, Duggan C, et al. Treating depression in general practice: factors affecting patients' treatment preferences. Br J Gen Pract. 2000; 50(460):905-6. [PubMed: 11141877]

Churchill 2001 . Churchill R, Hunot V, Corney R, Knapp M, McGuire H, Tylee A, et al. A systematic review of controlled trials of the effectiveness and cost-effectiveness of brief psychological treatments for depression. Health Technology Assessment. 2001; Vol. 5(issue 35): 1-173. [PubMed: 12387733]

Cipriani 2005 . Cipriani A, Brambilla P, Furukawa TA, Geddes J, Gregis M, Hotopf M, Malvini L, Barbui C. Fluoxetine versus other types of pharmacotherapy for depression. Cochrane Database of Systematic Reviews. 2005; (Issue 4) [DOI: 10.1002/14651858.CD004185.pub2].

Cipriani 2009 . Cipriani A, Santilli C, Furukawa TA, Signoretti A, Nakagawa A, McGuire H, et al. Escitalopram versus other antidepressive agents for depression. Cochrane Database of Systematic Reviews. 2009; (Issue 2) [DOI: 10.1002/14651858.CD006532.pub2].

Cipriani 2009a . Cipriani A, La Ferla T, Furukawa TA, Signoretti A, Nakagawa A, Churchill R, et al. Sertraline versus other antidepressive agents for depression. Cochrane Database of Systematic Reviews. 2009; (Issue 2) [DOI: 10.1002/14651858.CD006117.pub2].

Cipriani 2009b . Cipriani A, Furukawa TA, Salanti G, Geddes JR, Higgins JP, Churchill R, et al. Comparative efficacy and acceptability of 12 new-generation antidepressants: a multipletreatments meta-analysis. Lancet. 2009; 373(9665):746-58. [PubMed: 19185342]

Clark 1995 . Clark DA. Perceived limitations of standard cognitive therapy: A consideration of efforts to revise Beck's theory and therapy. Journal of Cognitive Psychotherapy: An International Quarterly. 1995; 9(3):1995.

Crits 1992 . Crits CP. The efficacy of brief dynamic psychotherapy: a meta-analysis. American Journal of Psychiatry. 1992; 149:151-8. [PubMed: 1531169]

Cuijpers 2002 . Cuijpers P, Smit F. Excess mortality in depression: a meta-analysis of community studies. Journal of Affective Disorders. 2002; 72:227-36. [PubMed: 12450639]

Cuijpers 2007 . Cuijpers P, van Straten A, Warmerdam L. Behavioral activation treatments of depression: a meta-analysis. Clinical Psychology Review. 2007; 27(3):318-26. [PubMed: 17184887]

Cuijpers 2008 . Cuijpers P, van Straten A, Andersson G, van Oppen P. Psychotherapy for depression in adults: a meta-analysis of comparative outcome studies. J Consult Clin Psychol. 2008; 76(6): 909-22. [PubMed: 19045960]

D'Zurilla 1971 . D'Zurilla TJ, Goldfried MR. Problem solving and behavior modification. Journal of Abnormal Psychology. 1971; 78(1):107-26. [PubMed: 4938262]

de Mello 2005 . de Mello MF, de Jesus MJ, Bacaltchuk J, Verdeli H, Neugebauer R. A systematic review of research findings on the efficacy of interpersonal therapy for depressive disorders. European Archives of Psychiatry and Clinical Neuroscience. 2005; 255(2):75-82. [PubMed: 15812600]

De Shazer 1988 . De Shazer, S. Clues: investigating solutions in brief therapy. WW Norton; New York: 1988.

Deeks 1997 . Deeks JJ. Are you sure that's a standard deviation? (part 1). Cochrane News. 1997; 10:11-2.

Deeks 2008 . Deeks, JJ.; Higgins, JPT.; Altman, DG.; On behalf of the Cochrane Statistical Methods Group. Chapter 9: Analysing data and undertaking meta-analyses. In: Higgins, JPT.; Green, S., editors. Cochrane Handbook for Systematic Reviews of Interventions. Version 5.0.2. The Cochrane Collaboration; 2008. Available from www.cochrane-handbook.org [updated September 2009]

Dobson 2001 . Dobson Keith, S. Handbook of cognitive-behavioral therapies. 2nd Edition. Guilford Press; New York; London: 2001.

Dunn 2007 . Dunn, M. Cognitive analytic therapy. In: Dryden, W., editor. In Dryden's Handbook of Individual Therapy. Sage; London: 2007. p. 584 
Egan 1986 . Egan, G. The skilled helper : a systematic approach to effective helping. 3rd Edition. Brooks/Cole Pub. Co; Monterey, California: 1986.

Ekers 2008 . Ekers D, Richards D, Gilbody S, Ekers D, Richards D, Gilbody S. A meta-analysis of randomized trials of behavioural treatment of depression. Psychological Medicine. 2008; 38(5): 611-23. [PubMed: 17903337]

Elkin 1989 . Elkin I, Shea MT, Watkins JT, Imber SD, Sotsky SM, Collins JF, et al. National Institute of Mental Health Treatment of Depression Collaborative Research Program: General Effectiveness of Treatments. Archives of General Psychiatry. 1989; 46(11):971-82. [PubMed: 2684085]

Ellis 1962 . Ellis, A. Reason and emotion in psychotherapy. Lyle Stuart; N.Y.: 1962.

Ellis 1979 . Ellis, A., editor. Theoretical and Empirical Foundations of Rational-Emotive Therapy. Brooks/Cole; Monterey: 1979.

Ellis 2004 . Ellis P. Australian and New Zealand clinical practice guidelines for the treatment of depression. Australian and New Zealand Journal of Psychiatry. 2004; 38(6):389-407. [PubMed: 15209830]

ESEMeD/MHEDEA 2004 . ESEMeD/MHEDEA 2000 investigators. Prevalence of mental disorders in Europe: results from the European Study of the Epidemiology of Mental Disorders (ESEMeD) project. Acta Psychiatrica Scandinavica. 2004; 109(s420):21-7.

Frank 1990 . Frank E, Kupfer DJ, Perel JM, Cornes C, Jarrett DB, Mallinger AG, et al. Three-Year Outcomes for Maintenance Therapies in Recurrent Depression. Archives of General Psychiatry. 1990; 47(12):1093-9. [PubMed: 2244793]

Freud 1949 . Freud, S. An outline of psychoanalysis. Hogarth Press; London: 1949.

Fuchs 1977 . Fuchs CZ, Rehm LP. Self-Control Behavior-Therapy Program for Depression. Journal of Consulting and Clinical Psychology. 1977; 45(2):206-15. [PubMed: 850005]

Furukawa 2005 . Furukawa TA, Cipriani A, Barbui C, Brambilla P, Watanabe N. Imputing response rates from means and standard deviations in meta-analysis. Internal Clinical Psychopharmacology. 2005; 20:49-52.

Furukawa 2006 . Furukawa TA, Barbui C, Cipriani A, Brambilla P, Watanabe N. Imputing missing standard deviations in meta-analyses can provide accurate results. Journal of Clinical Epidemiology. 2006; 59:7-10. [PubMed: 16360555]

Gaudiano 2008 . Gaudiano BA. Cognitive-behavioural therapies: achievements and challenges. Evidence-Based Mental Health. 2008; 11(1):5-7. [PubMed: 18223042]

Gloaguen 1998 . Gloaguen V, Cottraux J, Cucherat M, Blackburn IM, Gloaguen V, Cottraux J, et al. A meta-analysis of the effects of cognitive therapy in depressed patients. Journal of Affective Disorders. 1998; 49(1):59-72. [PubMed: 9574861]

Grant 2004 . Grant, A. Cognitive behavioural therapy in mental health care. SAGE; London: 2004.

Greenberg 1998 . Greenberg LS, Watson J. Experiential therapy of depression: Differential effects of client-centered relationship conditions and process experiential interventions. Psychotherapy Research. 1998; 8(2):210-24.

Guaiana 2007 . Guaiana G, Barbui C, Hotopf M. Amitriptyline for depression. Cochrane Database of Systematic Reviews. 2007; (Issue 3) [DOI: 10.1002/14651858.CD004186.pub2].

Guthrie 1999 . Guthrie E. Psychodynamic Interpersonal Therapy. Advances in Psychiatric Treatment. 1999; 5(2):135-45.

Guy 1976 . Guy, W. ECDEU Assessment Manual for Psychopharmacology-Revised. Vol. 218. NIMH Psychopharmacology Research Branch, Division of Extramural Research Programs; 1976. p. 22

Hamilton 1959 . Hamilton M. The assessment of anxiety states by rating. British Journal of Medical Psychology. 1959; 32(1):50-5. [PubMed: 13638508]

Hamilton 1960 . Hamilton M. A rating scale for depression. Journal of Neurology, Neurosurgery, and Psychiatry. 1960; 23:56-62.

Hamilton 1967 . Hamilton M. Development of a rating scale for primary depressive illness. British Journal of Social \& Clinical Psychology. 1967; 6(4):278-96. [PubMed: 6080235] 
Hayes 1999 . Hayes, SC.; Strosahl, K.; Wilson, KG. Acceptance and commitment therapy : an experiential approach to behavior change. Guilford Press; New York; London: 1999.

Hayes 2006 . Hayes SC, Luoma JB, Bond FW, Masuda A, Lillis J. Acceptance and commitment therapy: model, processes and outcomes. Behaviour research and therapy. 2006; 44(1):1-25. [PUBMED: 16300724]. [PubMed: 16300724]

Henken 2007 . Henken T, Huibers MJH, Churchill R, Restifo KK, Roelofs JJ. Family therapy for depression. Cochrane Database of Systematic Reviews. 2007; (Issue 3) [DOI: 10.1002/14651858.CD006728].

Higgins 2003 . Higgins JPT, Thompson SG, Deeks JJ, Altman DG. Measuring inconsistency in meta-analyses. BMJ. 2003; 327(7414):557-60. [PubMed: 12958120]

Higgins 2008 . Higgins, JPT.; Green, S., editors. Cochrane Handbook for Systematic Reviews of Interventions. Version 5.0.2. The Cochrane Collaboration; 2008. Available from www.cochranehandbook.org [updated September 2009]

Higgins 2008a . Higgins, JPT.; Altman, DG.; On behalf of the Cochrane Statistical Methods Group and the Cochrane Bias Methods Group. Assessing risk of bias in included studies. In: Higgins, JPT.; Green, S., editors. Cochrane Handbook for Systematic Reviews of Interventions. Version 5.0.2. The Cochrane Collaboration; 2008. Available from www.cochrane-handbook.org [updated September 2009]

Higgins 2008b . Higgins, JPT.; Deeks, JJ.; Altman, DG.; On behalf of the Cochrane Statistical Methods Group. Special topics in statistics. In: Higgins, JPT.; Green, S., editors. Cochrane Handbook for Systematic Reviews of Interventions. Version 5.0.2. The Cochrane Collaboration; 2008. Available from www.cochrane-handbook.org [updated September 2009]

Hobson 1985 . Hobson, RF. Forms of Feeling: The Heart of Psychotherapy. Tavistock Publications; London: 1985.

Hofmann 2008 . Hofmann SG, Asmundson GJG. Acceptance and mindfulness-based therapy: New wave or old hat? Clinical Psychology Review. 2008; 28(1):1-16. [PubMed: 17904260]

Hollanders 1999 . Hollanders H, McLeod J. Theoretical orientation and reported practice: A survey of eclecticism among counsellors in Britain. British Journal of Guidance \& Counselling. 1999; 27(3):405-14.

Hollanders 2007 . Hollanders, H. Integrative and eclectic approaches. In: Dryden, W., editor. In Dryden's Handbook of Individual Therapy. Sage; London: 2007. p. 584

Hopko 2003 . Hopko DR, Lejuez CW, Ruggiero KJ, Eifert GH. Contemporary behavioral activation treatments for depression: Procedures, principles, and progress. Clinical Psychology Review. 2003; 23(5):699-717. [PubMed: 12971906]

Horvath 1986 . Horvath, AO; Greenberg, L. The development of the Working Alliance Inventory: A research handbook. In: Greenberg, L.; Pinsoff, W., editors. Psychotherapeutic Processes: A Research Handbook. Guildford Press; New York: 1986.

Hunot 2007 . Hunot VM, Horne R, Leese MN, Churchill RC. A Cohort Study of Adherence to Antidepressants in Primary Care: The Influence of Antidepressant Concerns and Treatment Preferences. Prim Care Companion J Clin Psychiatry. 2007; 9(2):91-9. [PubMed: 17607330]

isIPT [ND] . isIPT. [accessed 29 April 2010] International Society for Interpersonal Psychotherapy. http://www.interpersonalpsychotherapy.org/http://www.interpersonalpsychotherapy.org/

Ivnik 1992 . Ivnik RJ, Malec JF, Smith GE, Tangalos EG, Petersen RC, Kokmen E. Mayo's older American normative studies: updated AVLT norms for age 56-97. The Clinical Neuropsychologist. 1992; 6(s1):8-103.

Jung 1963 . Jung, CG. Memories, Dreams, Reflections. Jaffé, A., editor. Collins and Routledge; London: 1963.

Kanfer 1970 . Kanfer, FH. Self-regulation: Research issues and speculations. In: Charles, Neuringer; Michael, Jack L., editors. Behavior Modification in Clinical Psychology. Appleton-CenturyCrofts; New York: 1970.

Keller 2000 . Keller MB, McCullough JP, Klein DN, Arnow B, Dunner DL, Gelenberg AJ, et al. A Comparison of Nefazodone, the Cognitive Behavioral-Analysis System of Psychotherapy, and their Combination for the Treatment of Chronic Depression. The New England Journal of Medicine. 2000; 342(20):1462-70. [PubMed: 10816183] 
Klein 1960 . Klein, M. Our Adult World and its Roots in Infancy. Tavistock; London: 1960.

Klerman 1984 . Klerman, GL.; Weissman, MM.; Rousaville, BJ.; Chevron, ES. Interpersonal psychotherapy for depression. Basic Books; New York: 1984.

Law 2003 . Law J, Garrett Z, Nye C. Speech and language therapy interventions for children with primary speech and language delay or disorder. Cochrane Database of Systematic Reviews. 2003; (Issue 3) [DOI: 10.1002/14651858.CD004110].

Lazarus 1971 . Lazarus, AA. Behavior therapy and beyond. McGraw; 1971.

Lazarus 1989 . Lazarus, AA. The practice of multimodal therapy: systematic, comprehensive, and effective psychotherapy. Johns Hopkins University Press; Baltimore: 1989.

Lazarus 2005 . Lazarus, AA. Multimodal therapy. 2nd Edition. Norcross, JC.; Goldfried, MR., editors. Oxford University Press; Oxford: 2005.

Leichsenring 2004 . Leichsenring F, Rabung S, Leibing E. The efficacy of short-term psychodynamic psychotherapy in specific psychiatric disorders: a meta-analysis. Archives of General Psychiatry. 2004; 61(12):1208-16. [PubMed: 15583112]

Lewinsohn 1972 . Lewinsohn PM, Libet J. Pleasant events, activity schedules, and depression. Journal of Abnormal Psychology. 1972; 79:291-95. [PubMed: 5033370]

Lewinsohn 1974 . Lewinsohn, PM. A Behavioural Approach to Depression. In: Friedman, RJ.; Katz, MM., editors. The psychology of depression: contemporary theory and research. Winston; Washington, D.C.: 1974. p. 157-78.

Lewinsohn 1984 . Lewinsohn, PM. The coping with depression course : a psychoeducational intervention for unipolar depression. Castalia Pub. Co.; Eugene, OR: 1984.

Llewelyn 2003 . Llewelyn S. Cognitive Analytic Therapy: time and process. Psychodynamic Practice: Individuals, Groups and Organisations. 2003; 9(4):501-20.

Lu 2004 . Lu G, Ades AE. Combination of direct and indirect evidence in mixed treatment comparisons. Statistics in Medicine. 2004; 23:3105-24. [PubMed: 15449338]

Luborsky 1962 . Luborsky L. Clinician's judgments of mental health. Arch Gen Psychiatry. 1962; 7:407-17. [PubMed: 13931376]

Luborsky 1984 . Luborsky, L. Principles of Psychoanalytic Psychotherapy: A Manual for Supportive/Expressive Treatment. Basic Books; New York: 1984.

Mahoney 1978 . Mahoney, MJ.; Arnkoff, DB. Cognitive and Self-Control Therapies. In: Garfield, SL.; Bergin, AE., editors. Handbook of psychotherapy and behavior change: an empirical analysis. 2nd Edition. Wiley; New York; Chichester: 1978.

Malan 1963 . Malan, DH. A Study of Brief Psychotherapy. Tavistock Publications; Charles C. Thomas; London: Springfield, Illinois: 1963. p. xivp. 312

Mann 1973 . Mann, J. Time-Limited Psychotherapy. Harvard University Press; Cambridge MA: 1973.

Mansell 2008 . Mansell W. The seven C's of CBT: A consideration of the future challenges for cognitive behaviour therapy. Behavioural and Cognitive Psychotherapy. Nov.2008 36(6)

Markowitz 1998 . Markowitz JC, Svartberg M, Swartz HA. Is IPT time-limited psychodynamic psychotherapy? The Journal of Psychotherapy Practice and Research. 1998; 7(3):185-95. [PubMed: 9631340]

Marks 1982 . Marks, IM. Cure and Care of Neuroses: Theory and Practice of Behavioural Psychotherapy. Wiley; New York: 1982.

Maslow 1943 . Maslow AH. A theory of human motivation. Psychological Review. 1943; 50:270_ 96.

May 1961 . May, R., editor. Existential psychology. Random House; New York: 1961.

McCullough 1984 . McCullough JP. Cognitive-behavioral analysis system of psychotherapy: An interactional treatment approach for dysthymic disorder. Psychiatry: Journal for the Study of Interpersonal Processes. 1984; 47(3):234-50.

McCullough 2003 . McCullough JP. Treatment for chronic depression: Cognitive behavioral analysis system of psychotherapy (CBASP). Journal of Psychotherapy Integration. 2003; 13(3-4):241-63.

McMurdo 2005 . McMurdo ME, Witham MD, Gillespie ND. Including older people in clinical research. BMJ. 2005; 331(7524):1036-7. [PubMed: 16269472] 
Mitchell 2009 . Mitchell AJ, Vaze A, Rao S. Clinical diagnosis of depression in primary care: a meta-analysis. The Lancet. 2009; 374(9690):609-19.

Montgomery 1979 . Montgomery SA, Asberg M. A new depression scale designed to be sensitive to change. British Journal of Psychiatry. 1979; 134:382-9. [PubMed: 444788]

Mykletun 2009 . Mykletun A, Bjerkeset O, Øverland S, Prince M, Dewey M, Stewart R. Levels of anxiety and depression as predictors of mortality: the HUNT study. British Journal of Psychiatry. 2009; 195:118-125. (2009). [PubMed: 19648541]

Nezu 1986 . Nezu AM. Efficacy of A Social Problem-Solving Therapy Approach for Unipolar Depression. Journal of Consulting and Clinical Psychology. 1986; 54(2):196-202. [PubMed: 3700806]

NICE 2009 . NICE. Clinical Guideline 09 and 91. National Institute for Clinical Excellence; London: 2009. Depression: Treatment management of depression in adults, including adults with a chronic physical health problem.

Norcross 2005 . Norcross, JC.; Goldfried, MR. Handbook of psychotherapy integration. 2nd Edition. Oxford University Press; Oxford: 2005.

O'Connell 2007 . O’Connell, B. Solution-focused therapy. In: Dryden, W., editor. In Dryden's Handbook of Individual Therapy. Sage; London: 2007.

Orlinsky 2005 . Orlinsky, DE.; Rønnestad, MH. How psychotherapists develop: a study of therapeutic work and professional growth. American Psychological Association; Washington DC: 2005.

Pilgrim 2002 . Pilgrim, D. The Cultural Context of British Psychotherapy. In: Dryden, W., editor. The Handbook of Individual Psychotherapy. 4th Edition. Sage; London: 2002.

Prochaska 1982 . Prochaska JO, DiClemente CC. Transtheoretical therapy: toward a more integrative model of change. Psychotherapy: Theory, Research \& Practice. 1982; 19(3):276-88.

Rachman 1997 . Rachman, SJ. The evolution of cognitive behaviour therapy. In: Clark, DM.; Fairburn, CG., editors. Science and practice of cognitive behaviour therapy. Oxford University Press; Oxford: 1997. p. 2-26.

Rait 2005 . Rait G, Fletcher A, Smeeth L, Brayne C, Stirling S, Nunes M, et al. Prevalence of cognitive impairment: results from the MRC trial of assessment and management of older people in the community. Age and Ageing. 2005; 34(3):242-8. [PubMed: 15863409]

Rait 2009 . Rait G, Walters K, Griffin M, Buszewicz M, Petersen I, Nazareth I. Recent trends in the incidence of recorded depression in primary care. The British Journal of Psychiatry. 2009; 195(6):520-4. [PubMed: 19949202]

Rehm 1977 . Rehm LP. Self-Control Model of Depression. Behavior Therapy. 1977; 8(5):787-804.

Riedel-Heller 2005 . Riedel-Heller SG, Matschinger H, Angermeyer MC. Mental disorders--who and what might help? Help-seeking and treatment preferences of the lay public. Soc Psychiatry Psychiatr Epidemiol. 2005; 40(2):167-74. [PubMed: 15685409]

Roca 2009 . Roca M, Gili M, Garcia-Garcia M, Salva J, Vives M, Garcia Campayo J, et al. Prevalence and comorbidity of common mental disorders in primary care. Journal of Affective Disorders. 2009; 119(1-3):52-8. [PubMed: 19361865]

Rogers 1951 . Rogers, C. Client-centered therapy: Its current practice, implications and theory. Constable; London: 1951.

Roth 2008 . Roth ADP. Using an evidence-based methodology to identify the competences required to deliver effective cognitive and behavioural therapy for depression and anxiety disorders. Behavioural and Cognitive Psychotherapy. Mar.2008 36(2)

Ryle 1979 . Ryle A. The focus in brief interpretive psychotherapy: dilemmas, traps and snags as target problems. The British Journal of Psychiatry. 1979; 134(1):46-54. [PubMed: 760923]

Ryle 1990 . Ryle, A. Cognitive-analytic Therapy - Active Participation in Change: New Integration in Brief Psychotherapy. John Wiley \& Sons; Chichester: 1990.

Shapiro 1990 . Shapiro, DA.; Startup, MJ. Raters' manual for the Sheffield psychotherapy rating scale. MRC/ESRC Social and Applied Psychology Unit, University of Sheffield; Sheffield: 1990.

Skinner 1953 . Skinner, BF. Science and human behaviour. Free Press; New York: 1953. 
Spielberger 1983 . Spielberger, CD.; Gorsuch, RL.; Lushene, R.; Vagg, PR.; Jacobs, GA. Manual for the State-Trait Anxiety Inventory. Consulting Psychologists Press; Palo Alto, CA: 1983.

Stiles 2008 . Stiles WB, Barkham M, Mellor-Clark J, Connell J. Effectiveness of cognitivebehavioural, person-centred, and psychodynamic therapies in UK primary-care routine practice: replication in a larger sample. Psychol Med. 2008; 38(5):677-88. [PubMed: 17825124]

Strupp 1984 . Strupp, H.; Binder, J. Psychotherapy in a New Key: A Guide to Time-Limited Dynamic Psychotherapy. Basic Books; New York: 1984.

Svartberg 1991 . Svartberg M, Stiles TC. Comparative effects of short-term psychodynamic psychotherapy: a meta-analysis. Journal of Consulting and Clinical Psychology. 1991; 59(5): 704-14. [PubMed: 1835464]

Trepper 2010 . Trepper, TS.; McCollum, EE.; De Jong, P.; Korman, H.; Gingerich, W.; Franklin, C. Solution Focused Therapy Treatment Manual for Working with Individuals. Research Committee of the Solution Focused Brief Therapy Association; 2010.

Ustun 2004 . Ustun TB, Yuso-Mateos JL, Chatterji S, Mathers C, Murray CJ. Global burden of depressive disorders in the year 2000. Br J Psychiatry. 2004; 184:386-92. [PubMed: 15123501]

van Geffen 2009 . van Geffen EC, Gardarsdottir H, van Hulten R, van Dijk L, Egberts AC, Heerdink ER. Initiation of antidepressant therapy: do patients follow the GP's prescription? Br J Gen Pract. 2009; 59(559):81-7. [PubMed: 19192372]

Wampold 2002 . Wampold BE, Minami T, Baskin TW, Callen TS. A meta-(re)analysis of the effects of cognitive therapy versus 'other therapies' for depression. Journal of Affective Disorders. 2002; 68(2-3):159-65. [PubMed: 12063144]

Ware 1993 . Ware, JE.; Snow, KK.; Kosinski, M.; Gandek, B. SF-36 Health Survey: Manual and interpretation guide. Health Institute, New England Medical Center; Boston, MA: 1993.

Watanabe 2007 . Watanabe N, Hunot V, Omori IM, Churchill R, Furukawa TA. Psychotherapy for depression among children and adolescents: a systematic review. Acta Psychiatr Scand. 2007; 116(2):84-95. [PubMed: 17650269]

Watson 1924 . Watson, JB. Behaviorism. Norton; New York: 1924.

Watzlavick 1974 . Watzlavick, P.; Weakland, J.; Fisch, R. Change: principles of problem formation and problem resolution. W W Norton; New York: 1974. Brief Problem SolvingChange: principles of problem formation and problem resolution. New York: W W Norton.

Weissman 2007 . Weissman, MM.; Markowitz, JC.; Klerman, GL. Clinician's quick guide to interpersonal psychotherapy. Oxford University Press; New York; Oxford: 2007.

Welton 2009 . Welton NJ, Caldwell DM, Adamopoulos E, Vedhara K. Mixed treatment comparison meta-analysis of complex interventions: psychological interventions in coronary heart disease. American Journal of Epidemiology. 2009; 169(9):1158-65. [PubMed: 19258485]

WHO 1978 . World Health Organisation (WHO). The Ninth Revision of the International Classification of Diseases and Related Health Problems (ICD-9). WHO; Geneva: 1978.

WHO 1992 . World Health Organisation (WHO). The ICD-10 Classification of Mental and Behavioural Disorders. WHO; Geneva: 1992.

WHO 2001 . World Health Organisation (WHO). World Health Report 2001: Mental Health: New Understanding, New Hope. WHO; Geneva: 2001.

WHO 2004 . WHO. The global burden of disease: 2004 update. WHO; Geneva: 2004.

WHO 2008 .WHO. 2008. http://www.who.int/healthinfo/global_burden_disease/projections/en/ index.htmlhttp://www.who.int/healthinfo/global_burden_disease/projections/en/index.html

WHOQL 1998 . WHO. The World Health Organisation Quality of Life Assessment (WHOQOL): development and general psychometric properties. Social Science and Medicine. 1998; 46:156985. [PubMed: 9672396]

Williams 1997 . Williams, JMG. Specific problems and disorders: Depression. In: Clark, DA.; Fairburn, CG., editors. Science and Practice of Cognitive Behaviour Therapy. Oxford University Press; Oxford: 1997.

Wilson 2008 . Wilson KC, Mottram PG, Vassilas CA. Psychotherapeutic treatments for older depressed people. Cochrane Database of Systematic Reviews. 2008; (Issue 1) [DOI: 10.1002/14651858.CD004853.pub2.]. 
Wing 1994 . Wing, J. Outcomes into Clinical Practice. BMJ publishing; London: 1994. Measuring mental health outcomes: a perspective from the Royal College of Psychiatrists.

Wolpe 1958 . Wolpe, J. Psychotherapy by Reciprocal Inhibition. Stanford University Press; Witwatersrand University Press; Standford: Johannesburg: 1958.

Yalom 2001 . Yalom, ID. The gift of therapy: Reflections on being a therapist. Piatkus; London: 2001.

* Indicates the major publication for the study 